\title{
Fractional Liouville equation on lattice phase-space
}

\author{
Vasily E. Tarasov \\ Skobeltsyn Institute of Nuclear Physics, Lomonosov Moscow State University, Moscow 119991, Russia
}

\section{H I G H L I G H T S}

- Liouville equation for lattice phase-space with long-range jumps is suggested.

- Continuous limit of lattice Liouville equation gives fractional Liouville equation.

- Fractional Liouville equation is used to describe media with power-law nonlocality.

\section{A R T I C L E I N F O}

\section{Article history:}

Received 27 August 2014

Received in revised form 10 November 2014

Available online 27 November 2014

\section{Keywords:}

Liouville equation

Fractional equation

Fractional derivative

Lattice

Long-range jump

\begin{abstract}
A B S T R A C T
In this paper we propose a lattice analog of phase-space fractional Liouville equation. The Liouville equation for phase-space lattice with long-range jumps of power-law types is suggested. We prove that the continuum limit transforms this lattice equation into Liouville equation with conjugate Riesz fractional derivatives of non-integer orders with respect to coordinates of continuum phase-space. An application of the fractional Liouville equation with these Riesz fractional derivatives to describe properties of plasma-like nonlocal media is considered.
\end{abstract}

(C) 2014 Elsevier B.V. All rights reserved.

\section{Introduction}

A consistent formulation of the nonlocal statistical mechanics was first constructed by Vlasov in Refs. [1,2]. Fractional calculus [3-9] has a lot of applications in physics [10-16] and it allows us to take into account fractional power-law nonlocality of continuously distributed systems. Using the fractional calculus, we can consider fractional differential equations for conservation of probability in generalized phase spaces. The Liouville equation with phase-space fractional derivatives allows us to formulate fractional statistical mechanics that describes systems with power-law nonlocality. The fractional statistical mechanics can be considered as a special form of the nonlocal statistical mechanics [13]. It should be noted that the use of the fractional derivative of non-integer order is actually equivalent to using an infinite number of derivatives of integer orders, which can be arbitrarily large values (see Lemma 15.3 in Ref. [3]).

We note that fractional Liouville and Bogoliubov equations are discussed in Refs. [17-23]. There are two different types of approaches to space-fractional generalization of the Liouville equation:

(1) The first approach to generalization of the Liouville equation in the framework of fractional dynamics has been suggested in Refs. [17-20]. This generalization is derived from the normalization condition with the fractional integration over phase-space coordinates. This fractional normalization condition is interpreted as an equation in fractional-dimensional phase-space or in phase-space with fractional powers of phase-space coordinates. We should note that the fractional Liouville equations, which are suggested in Refs. [17-20], do not contain fractional derivatives of non-integer orders.

E-mail address: tarasov@theory.sinp.msu.ru. 
(2) The second approach, which is based on the Liouville equation with fractional-order derivatives, has been suggested in Refs. [21,22,13], where the Caputo type of fractional derivatives is used. To obtain this equation, we use the conservation of probability for a fractional differential volume element of the phase space. The suggested fractional Liouville equation is used to derive the fractional kinetics equations. The Bogoliubov hierarchy equations with fractional derivatives with respect to phase space coordinates are derived in Refs. [21,22]. The Vlasov equation and the Fokker-Planck equation with the Caputo fractional derivatives are also obtained from the fractional Liouville equations. In Ref. [23] the Liouville equation with the Caputo fractional derivatives is used to describe media with spatial dispersion of power-law type that is considered in Ref. [24]. We should note that this phase-space fractional Liouville equation is considered only for Caputo fractional derivatives.

As it was shown in Refs. [25-27] (see also Refs. [28-38]), the continuum equations with fractional derivatives can be directly connected to lattice models with long-range properties. Long-range interaction and properties are important for different problems in statistical mechanics [39-41], in kinetic theory and non-equilibrium statistical mechanics [42,43], in the theory of non-equilibrium phase transitions $[44,45]$. A connection between the dynamics of lattice system with long-range properties and the fractional continuum equations is proved by using the special transform operation [25-27] and it has been applied to different subjects [32-38].

In this paper, we propose the Liouville equation for lattice phase-space. This Liouville equation describes fractional dynamics of a distribution function on an unbounded homogeneous lattice with long-range jumps from one site to other sites. We prove that continuous limit of the suggested lattice Liouville equation gives the fractional Liouville equation for continuum phase-space. This fractional Liouville equation contains generalized conjugate Riesz derivatives of non-integer orders with respect to coordinates and momenta. As an example, we consider an application of the fractional Liouville equation with these Riesz derivatives to describe properties of plasma-like nonlocal media.

\section{Liouville equation for lattice with long-range jumps}

In statistical mechanics [46], the basic concept is the ensemble that is a set of classical systems identical in nature, which are subjected to forces determined by identical laws [47], but distributed in phase-space. We can state that the statistical ensemble is a set of independent systems identical in equations of motion, but differing in their initial conditions [48]. It is usually assumed that the systems are continuously distributed in the phase-space. In this paper, we consider systems of particles that are distributed in lattice phase-space. Note that the statistical method should be used not only for systems with a very large number of particles. It is necessary to use this method in every case, even that of a single particle in the simplest possible conditions [49]. In the Liouvillian picture, we can describe the dynamics of a statistical ensemble in the phase-space points (sites) through which the ensemble points move. In this case, properties of the ensemble are assigned to points (sites) in phase-space at each given time, without an attempt to identify the individual system of the ensemble. In this paper, we consider a statistical ensemble on the lattice phase-space.

Let us consider a system of classical identical $N$ spinless point particles, which are characterized by the phase-space coordinates $q_{s, k}$ and $p_{s, k}$ with $s=1, \ldots, N, k=1,2,3$. As a lattice analog of continuum phase-space for this system we will use an unbounded lattice in $6 N$-dimensional Euclidean phase-space $\mathbb{R}^{6 N}$. The lattice is characterized by space periodicity. For unbounded phase-space lattice we define $6 N$ non-coplanar vectors $\mathbf{a}_{1}, \ldots, \mathbf{a}_{6 N}$, that are the shortest vectors by which a lattice can be displaced such that it is brought back into itself. For simplification, we consider a lattice with mutually perpendicular lattice vectors $\mathbf{a}_{j}$. We choose the Cartesian coordinate system such that the directions of the axes coincide with the vector $\mathbf{a}_{j}$, such that $\mathbf{e}_{j}=\mathbf{a}_{j} /\left|\mathbf{a}_{j}\right|$ are the basis vectors of the Cartesian coordinate system in $\mathbb{R}^{6 N}$. This simplification means that we consider $6 \mathrm{~N}$-dimensional analog of the primitive orthorhombic Bravais lattice.

Choosing the coordinate origin at one of the sites, all phase-space lattice sites can be numbered by the vectors $\mathbf{n}_{q}=$ $\left(n_{1}, \ldots, n_{3 N}\right)$ and $\mathbf{n}_{p}=\left(n_{3 N+1}, \ldots, n_{6 N}\right)$, where $n_{j}(j=1, \ldots, 6 N)$ are integer. The position of a lattice site is defined by the vector

$$
\mathbf{x}(\mathbf{n})=\mathbf{x}\left(\mathbf{n}_{q}\right)+\mathbf{x}\left(\mathbf{n}_{p}\right)=\sum_{j=1}^{6 N} n_{j} \mathbf{a}_{j},
$$

where

$$
\mathbf{x}\left(\mathbf{n}_{q}\right)=\sum_{j=1}^{3 N} n_{j} \mathbf{a}_{j}, \quad \mathbf{x}\left(\mathbf{n}_{p}\right)=\sum_{j=3 N+1}^{6 N} n_{j} \mathbf{a}_{j} .
$$

Considering the statistical ensemble on the phase-space lattice, the $N$-particle system is represented by a point-particle, which is moved through the lattice sites. This lattice particle, which is a lattice analog of the $N$-particle system, will be called the lattice quasi-particle. We assume that it can be localized in the sites of the phase-space lattice, i.e. the positions of the quasi-particle coincide with the lattice sites. Then the vectors $\mathbf{n}_{q}$ and $\mathbf{n}_{p}$ can be used to describe the quasi-particle. We will consider long-range jumps of the quasi-particle on the lattice phase-space. 
The distribution function of lattice quasi-particle, which is the probability density to be in the lattice site, will be denoted by $\rho\left(\mathbf{n}_{q}, \mathbf{n}_{p}, t\right)=\rho\left(n_{1}, \ldots, n_{6 N}, t\right)$, where the vectors $\mathbf{n}_{q}$ and $\mathbf{n}_{p}$ define the site. We can use the notation $\rho_{L}\left(\mathbf{n}_{q}, \mathbf{n}_{p}, t\right)$ instead of $\rho\left(\mathbf{n}_{q}, \mathbf{n}_{p}, t\right)$ to emphasize that it is a lattice function. The function $\rho\left(\mathbf{n}_{q}, \mathbf{n}_{p}, t\right)$ satisfies the conditions

$$
\sum_{n_{1}=-\infty}^{+\infty} \ldots \sum_{n_{6 N}=-\infty}^{+\infty} \rho\left(n_{1}, \ldots, n_{6 N}, t\right)=1, \quad \rho\left(n_{1}, \ldots, n_{6 N}, t\right) \geq 0(t \in \mathbb{R}) .
$$

In the lattice, any particle of the $N$-particle system is described by the six numbers

$$
\mathbf{n}(s)=\left(n_{s}, n_{s+1}, n_{s+2}, n_{3 N+s}, n_{3 N+s+1}, n_{3 N+s+2}\right), \quad(s=1, \ldots, N) .
$$

Since we assume that the system consists of the identical $N$ particles, the function $\rho\left(n_{1}, \ldots, n_{6 N}, t\right)$ has the symmetry with respect to permutations $\mathbf{n}(s) \longleftrightarrow \mathbf{n}\left(s^{\prime}\right)$, where $1 \leq s, s^{\prime} \leq N$. We also can define reduced $s$-particle distribution functions by the equation

$$
\rho_{s}\left(\mathbf{n}_{q}, \mathbf{n}_{p}, t\right)=\sum_{\mathbf{n}(s+1)} \ldots \sum_{\mathbf{n}(N)} \rho\left(\mathbf{n}_{q}, \mathbf{n}_{p}, t\right),
$$

where $\rho_{s}\left(\mathbf{n}_{q}, \mathbf{n}_{p}, t\right)$ depends on the vectors $\mathbf{n}(1), \ldots \mathbf{n}(s)$ only. In the case $s=N$, we can see that $\rho_{N}\left(\mathbf{n}_{q}, \mathbf{n}_{p}, t\right)=\rho\left(\mathbf{n}_{q}, \mathbf{n}_{p}, t\right)$.

Let us consider the equation for distribution function on unbounded homogeneous phase-space lattice in the form

$$
\begin{aligned}
& \frac{\partial \rho\left(\mathbf{n}_{q}, \mathbf{n}_{p}, t\right)}{\partial t}+\sum_{j=1}^{3 N} \mathbb{D}_{L, q}^{-}\left[\begin{array}{c}
\alpha_{j} \\
j
\end{array}\right]\left(V_{j}\left(\mathbf{m}_{q}, \mathbf{m}_{p}, t\right) \rho\left(\mathbf{m}_{q}, \mathbf{m}_{p}, t\right)\right) \\
& +\sum_{j=3 N+1}^{6 N} \mathbb{D}_{L, p}^{-}\left[\begin{array}{c}
\beta_{j} \\
j
\end{array}\right]\left(F_{j}\left(\mathbf{m}_{q}, \mathbf{m}_{p}, t\right) \rho\left(\mathbf{m}_{q}, \mathbf{m}_{p}, t\right)\right)=J\left(\mathbf{n}_{q}, \mathbf{n}_{p}, t\right),
\end{aligned}
$$

where the lattice function $\rho\left(\mathbf{n}_{q}, \mathbf{n}_{p}, t\right)$ is the probability density to find the quasi-particle at site ( $\left.\mathbf{n}_{q}, \mathbf{n}_{p}\right)$ at time $t$. In Eq. (6), the term $J\left(\mathbf{n}_{q}, \mathbf{n}_{p}, t\right)$ describes an external source. The lattice operators $\mathbb{D}_{L, q}^{-}\left[\begin{array}{c}\alpha_{j} \\ j\end{array}\right]$ and $\mathbb{D}_{L, p}^{-}\left[\begin{array}{c}\beta_{j} \\ j\end{array}\right]$ are defined by

$$
\mathbb{D}_{L}^{-}\left[\begin{array}{c}
\alpha_{j} \\
j
\end{array}\right] f\left(\mathbf{m}_{q}, \mathbf{m}_{p}, t\right)=\frac{1}{a_{j}^{\alpha_{j}}} \sum_{m_{j}=-\infty}^{+\infty} K_{\alpha_{j}}^{-}\left(n_{j}-m_{j}\right) f\left(\mathbf{m}_{q}, \mathbf{m}_{p}, t\right),
$$

where we have $\mathbb{D}_{L}^{-}\left[\begin{array}{c}\alpha_{j} \\ j\end{array}\right]=\mathbb{D}_{L, q}^{-}\left[\begin{array}{c}\alpha_{j} \\ j\end{array}\right]$ for $j=1, \ldots, 3 N$ and $\mathbb{D}_{L}^{-}\left[\begin{array}{c}\alpha_{j} \\ j\end{array}\right]=\mathbb{D}_{L, p}^{-}\left[\begin{array}{c}\beta_{j} \\ j\end{array}\right]$ with $\beta_{j}=\alpha_{j}$ for $j=3 N+1, \ldots, 6 N$. The kernel $K_{\alpha_{j}}^{-}\left(n_{j}-m_{j}\right)$ of the operator (7) describes the quasi-particle long-range jumps with length $n_{j}-m_{j}$ on the phase-space lattice. If $K_{\alpha_{j}}^{-}\left(n_{j}-m_{j}\right)>0$, then the kernel characterizes the jump to the site with $n_{j}$ from all other sites with $m_{j} \neq n_{j}$. If $K_{\alpha_{j}}^{-}\left(n_{j}-m_{j}\right)<0$, then the kernel describes the reverse process. In Eq. (6), the parameters $\alpha_{j}$ (and $\beta_{j}$ ) are positive real numbers that characterize how quickly the intensity of the jumps in the lattice decrease with increasing the jump length $\left|n_{j}-m_{j}\right|$. These parameters also can be considered as degrees of the power-law spatial dispersion in the lattice [32,34]. Eq. (6) is the lattice fractional Liouville equation that describes long-range jumps on $6 \mathrm{~N}$-dimensional phase-space lattice.

Let us consider the kernel $K_{\alpha_{j}}^{-}\left(n_{j}-m_{j}\right)$ in the form

$$
K_{\alpha_{j}}^{-}\left(n_{j}-m_{j}\right)=-\frac{\pi^{\alpha_{j}+1}\left(n_{j}-m_{j}\right)}{\alpha_{j}+2}{ }_{1} F_{2}\left(\frac{\alpha_{j}+2}{2} ; \frac{3}{2}, \frac{\alpha_{j}+4}{2} ;-\frac{\pi^{2}\left(n_{j}-m_{j}\right)^{2}}{4}\right), \quad \alpha_{j}>-2,
$$

where ${ }_{1} F_{2}$ is the Gauss hypergeometric function [50]. Note that expression (8) can be used not only for $\alpha_{j}>0$, but also for some negative values of $-2<\alpha_{j}<0$. In the kernel notation, the minus is used to mark that it is the odd functions of integer variable $n \in \mathbb{Z}$ such that $K_{\alpha_{j}}^{-}\left(n_{j}-m_{j}\right)=-K_{\alpha_{j}}^{-}\left(m_{j}-n_{j}\right)$ for all $n_{j}, m_{j} \in \mathbb{Z}$ and $j=1, \ldots, 6 N$.

The discrete Fourier transform

$$
\hat{K}_{\alpha_{j}}^{-}\left(k_{j}\right)=\sum_{n_{j}=-\infty}^{+\infty} \mathrm{e}^{-\mathrm{i} k_{j} n_{j}} K_{\alpha_{j}}^{-}\left(n_{j}\right)=-2 \mathrm{i} \sum_{n_{j}=1}^{\infty} K_{\alpha_{j}}^{-}\left(n_{j}\right) \sin \left(k_{j} n_{j}\right)
$$

of the kernels $K_{\alpha_{j}}^{-}\left(n_{j}\right)$ defined by (8) has the form

$$
\hat{K}_{\alpha_{j}}^{-}\left(k_{j}\right)=i \operatorname{sgn}\left(k_{j}\right)\left|k_{j}\right|^{\alpha_{j}} .
$$

Note that the function (8) can obtained by the equation

$$
K_{\alpha_{j}}^{-}(n)=-\frac{1}{\pi} \int_{0}^{\pi} k^{\alpha_{j}} \sin (n k) \mathrm{d} k
$$


that is the inverse relation to Eq. (9). For integer values of $\alpha_{j}$, we can get simpler equations instead of (8). For example, we have

$$
K_{1}^{-}(n)=\frac{(-1)^{n}}{n}, \quad K_{2}^{-}(n)=\frac{(-1)^{n} \pi}{n}+\frac{2\left(1-(-1)^{n}\right)}{\pi n^{3}}, \quad K_{3}^{-}(n)=\frac{(-1)^{n} \pi^{2}}{n}-\frac{6(-1)^{n}}{n^{3}},
$$

where $\left(1-(-1)^{n}\right)=2$ for odd $n$, and $\left((-1)^{n}-1\right)=0$ for even $n$.

In the general case, we can consider the kernels

$$
\hat{K}_{\alpha_{j}}^{-}\left(k_{j}\right)=i \operatorname{sgn}\left(k_{j}\right)\left|k_{j}\right|^{\alpha_{j}}+o\left(\left|k_{j}\right|^{\alpha_{j}}\right), \quad\left(k_{j} \rightarrow 0\right),
$$

where the little-o notation $o\left(\left|k_{j}\right|^{\alpha_{j}}\right)$ means the terms that include higher powers of $\left|k_{j}\right|$ than $\left|k_{j}\right|^{\alpha_{j}}$. The form (13) means that we consider lattices with weak spatial dispersion [32]. If we use condition (13) instead of (10), then we can consider wider class of kernels to describe the long-range lattice jumps. As an example of the kernel with (13), we give

$$
K_{\alpha_{j}}^{-}(n)=\frac{(-1)^{(n+1) / 2}(2[(n+1) / 2]-n) \Gamma\left(\alpha_{j}+1\right)}{2^{\alpha_{j}} \Gamma\left(\left(\alpha_{j}+n\right) / 2+1\right) \Gamma\left(\left(\alpha_{j}-n\right) / 2+1\right)},
$$

where the brackets [ ] mean the integral part, i.e., the floor function that maps a real number to the largest previous integer number. The expression $(2[(n+1) / 2]-n)$ is equal to zero for even $n=2 m$, and it is equal to 1 for odd $n=2 m-1$. Note that the kernel (14) is a real valued function since we have zero, when the expression $(-1)^{(n+1) / 2}$ becomes a complex number. It is easy to see that we can use Eq. (14) for all integer values $n \in \mathbb{Z}$.

For a wide class of physical $N$-particles systems, we can use

$$
V_{j}\left(\mathbf{m}_{q}, \mathbf{m}_{p}, t\right)=V_{j}\left(\mathbf{m}_{p}\right), \quad F_{j}\left(\mathbf{m}_{q}, \mathbf{m}_{p}, t\right)=F_{j}\left(\mathbf{m}_{q}, t\right) .
$$

These conditions mean that $V_{j}$ are components of the lattice analog of the particle velocity vector, and $F_{j}$ are components of the lattice analog of the force vector that is independent of the particle momenta. In this case, the Liouville equation (6) can be rewritten in the form

$$
\frac{\partial \rho\left(\mathbf{n}_{q}, \mathbf{n}_{p}, t\right)}{\partial t}+\sum_{j=1}^{3 N} V_{j}\left(\mathbf{m}_{p}\right) \mathbb{D}_{L, q}^{-}\left[\begin{array}{c}
\alpha_{j} \\
j
\end{array}\right] \rho\left(\mathbf{m}_{q}, \mathbf{m}_{p}, t\right)+\sum_{j=3 N+1}^{6 N} F_{j}\left(\mathbf{m}_{q}, t\right) \mathbb{D}_{L, p}^{-}\left[\begin{array}{c}
\beta_{j} \\
j
\end{array}\right] \rho\left(\mathbf{m}_{q}, \mathbf{m}_{p}, t\right)=J\left(\mathbf{n}_{q}, \mathbf{n}_{p}, t\right) .
$$

In the general case, the usual Leibniz rule for the lattice fractional derivative $\mathbb{D}_{L}^{-}$does not hold if $\alpha_{j} \neq 1$. This means that we have the inequality

$$
\mathbb{D}_{L, q}^{-}\left[\begin{array}{c}
\alpha_{j} \\
j
\end{array}\right]\left(f\left(\mathbf{m}_{q}\right) g\left(\mathbf{m}_{q}\right)\right) \neq f\left(\mathbf{m}_{q}\right) \mathbb{D}_{L, q}^{-}\left[\begin{array}{c}
\alpha_{j} \\
j
\end{array}\right] g\left(\mathbf{m}_{q}\right)+g\left(\mathbf{m}_{q}\right) \mathbb{D}_{L, q}^{-}\left[\begin{array}{c}
\alpha_{j} \\
j
\end{array}\right] f\left(\mathbf{m}_{q}\right) .
$$

This property is analogous to the characteristic property of derivatives of all fractional orders and all integer orders $\alpha_{j} \neq 1[51]$.

\section{Fractional Liouville equation for phase-space continuum}

In this section, we use the methods suggested in Refs. [25-27,13] to derive a fractional Liouville equation for phase-space continuum with power-law non-localities.

Let us define a transform operation that allows us to derive the fractional Liouville equation for continuum phase-space from the Liouville equation for lattice phase-space (6).

Definition. The lattice-continuum transform operation $\widetilde{\mathcal{T}}_{L \rightarrow C}$ is the combination

$$
\mathcal{T}_{L \rightarrow C}=\mathcal{F}^{-1} \circ \operatorname{Lim} \circ \mathcal{F}_{\Delta}
$$

of the following three operations:

(1) The operation $\mathcal{F}_{\Delta}$ is the discrete Fourier transform $f_{L}\left(\mathbf{n}_{q}, \mathbf{n}_{p}\right) \rightarrow \mathcal{F}_{\Delta}\left\{f_{L}\left(\mathbf{n}_{q}, \mathbf{n}_{p}\right)\right\}=\hat{f}\left(\mathbf{k}_{q}, \mathbf{k}_{p}\right)$ of the lattice function $f\left(\mathbf{n}_{q}, \mathbf{n}_{p}\right)$ that is defined by

$$
\hat{f}\left(\mathbf{k}_{q}, \mathbf{k}_{p}\right)=\mathcal{F}_{\Delta}\left\{f_{L}\left(\mathbf{n}_{q}, \mathbf{n}_{p}\right)\right\}=\sum_{n_{1}=-\infty}^{+\infty} \ldots \sum_{n_{6 N}=-\infty}^{+\infty} f_{L}\left(\left(\mathbf{n}_{q}, \mathbf{n}_{p}\right)\right) \mathrm{e}^{-i(\mathbf{k}, \mathbf{x}(\mathbf{n}))},
$$

where $(\mathbf{k}, \mathbf{x}(\mathbf{n}))=\left(\mathbf{k}_{q}, \mathbf{x}\left(\mathbf{n}_{q}\right)\right)+\left(\mathbf{k}_{p}, \mathbf{x}\left(\mathbf{n}_{p}\right)\right)$,

$$
\mathbf{x}(\mathbf{n})=\sum_{j=1}^{6 N} n_{j} \mathbf{a}_{j}, \quad \mathbf{x}\left(\mathbf{n}_{q}\right)=\sum_{j=1}^{3 N} n_{j} \mathbf{a}_{j}, \quad \mathbf{x}\left(\mathbf{n}_{p}\right)=\sum_{j=3 N+1}^{6 N} n_{j} \mathbf{a}_{j},
$$

and $a_{j}=2 \pi / k_{j 0}$ is distance between lattice particle in the direction $\mathbf{a}_{j}$. Eq. (19) means that we consider the lattice function $f_{L}\left(\mathbf{n}_{q}, \mathbf{n}_{p}\right)$ as discrete Fourier coefficients of some function $\hat{f}\left(\mathbf{k}_{q}, \mathbf{k}_{p}\right)$ for $k_{j} \in\left[-k_{j 0} / 2, k_{j 0} / 2\right]$, where $j=1, \ldots, 6 N$. 
(2) The operation Lim is the passage to the $\operatorname{limit} \hat{f}\left(\mathbf{k}_{q}, \mathbf{k}_{p}\right) \rightarrow \operatorname{Lim}\left\{\hat{f}\left(\mathbf{k}_{q}, \mathbf{k}_{p}\right)\right\}=\tilde{f}\left(\mathbf{k}_{q}, \mathbf{k}_{p}\right)$, where we use $a_{j} \rightarrow 0$ (or $k_{j 0} \rightarrow$ $\infty)$. It allows us to derive the function $\tilde{f}\left(\mathbf{k}_{q}, \mathbf{k}_{p}\right)$ from $\hat{f}\left(\mathbf{k}_{q}, \mathbf{k}_{p}\right)$, where $\tilde{f}\left(\mathbf{k}_{q}, \mathbf{k}_{p}\right)$ is the Fourier integral transform of the continuum function $f_{C}(\mathbf{q}, \mathbf{p})$, and $\hat{f}\left(\mathbf{k}_{q}, \mathbf{k}_{p}\right)$ is the discrete Fourier transform of the lattice function $f_{L}\left(\mathbf{n}_{q}, \mathbf{n}_{p}\right)$, where

$$
f_{L}\left(\mathbf{n}_{q}, \mathbf{n}_{p}\right)=\left(\prod_{j=1}^{6 N}\left(2 \pi / k_{j 0}\right)\right) f_{C}\left(\mathbf{x}\left(\mathbf{n}_{q}\right), \mathbf{x}\left(\mathbf{n}_{p}\right)\right),
$$

and $n_{j} a_{j}=2 \pi n_{j} / k_{j 0} \rightarrow q_{j}$ for $j=1, \ldots, 3 N$ and $n_{j} a_{j}=2 \pi n_{j} / k_{j 0} \rightarrow p_{j}$ for $j=3 N+1, \ldots, 6 N$.

(3) The operation $\mathcal{F}^{-1}$ is the inverse integral Fourier transform $\tilde{f}\left(\mathbf{k}_{q}, \mathbf{k}_{p}\right) \rightarrow \mathcal{F}^{-1}\left\{\tilde{f}\left(\mathbf{k}_{q}, \mathbf{k}_{p}\right)\right\}=f_{C}(\mathbf{q}, \mathbf{p})$ that is defined by

$$
f_{C}(\mathbf{q}, \mathbf{p})=\mathcal{F}^{-1}\left\{\tilde{f}\left(\mathbf{k}_{q}, \mathbf{k}_{p}\right)\right\}=\frac{1}{(2 \pi)^{6 N}} \int_{-\infty}^{+\infty} \mathrm{d} k_{1} \ldots \int_{-\infty}^{+\infty} \mathrm{d} k_{6 N} \mathrm{e}^{\mathrm{i} \sum_{j=1}^{6 N} k_{j} x_{j}} \tilde{f}\left(\mathbf{k}_{q}, \mathbf{k}_{p}\right) .
$$

For simplification, we will use notations $f\left(\mathbf{n}_{\mathbf{q}}, \mathbf{n}_{\mathbf{p}}\right)$ for $f_{L}\left(\mathbf{n}_{\mathbf{q}}, \mathbf{n}_{\mathbf{p}}\right)$ and $f(\mathbf{q}, \mathbf{p})$ for $f_{C}(\mathbf{q}, \mathbf{p})$. From the context it will be clear which function is considered.

We use the lattice-continuum transform operation $\widetilde{T}_{L \rightarrow C}$ for the lattice functions $f\left(\mathbf{m}_{q}, \mathbf{m}_{p}, t\right)$, for the product of two lattice functions $\left(f\left(\mathbf{m}_{q}, \mathbf{m}_{p}, t\right) g\left(\mathbf{m}_{q}, \mathbf{m}_{p}, t\right)\right)$ and for lattice fractional derivatives of the functions, i.e. $g\left(\mathbf{n}_{q}, \mathbf{n}_{p}, t\right)=$ $\mathbb{D}_{L}^{-}\left[\begin{array}{c}\alpha_{j} \\ j\end{array}\right] f\left(\mathbf{m}_{q}, \mathbf{m}_{p}, t\right)$. The operation $\mathcal{T}_{L \rightarrow C}$ can be applied not only for lattice functions but also for lattice operators. The operation $\mathcal{T}_{L \rightarrow C}$ allows us to map of lattice derivative $\mathbb{D}_{L}^{-}\left[\begin{array}{c}\alpha_{j} \\ j\end{array}\right]$ into a phase-space continuum fractional derivative $\mathbb{D}_{C}^{-}\left[\begin{array}{c}\alpha_{j} \\ j\end{array}\right]$ that is defined in the Appendix.

Proposition. The lattice-continuum transform operation $\widetilde{T}_{L \rightarrow C}$ maps the fractional Liouville equation for phase-space lattice (6) with the lattice operators (7) into the fractional Liouville equation for phase-space continuum

$$
\frac{\partial \rho(\mathbf{q}, \mathbf{p}, t)}{\partial t}+\sum_{j=1}^{3 N} \mathbb{D}_{C, q}^{-}\left[\begin{array}{c}
\alpha_{j} \\
j
\end{array}\right]\left(V_{j}(\mathbf{q}, \mathbf{p}, t) \rho(\mathbf{q}, \mathbf{p}, t)\right)+\sum_{j=3 N+1}^{6 N} \mathbb{D}_{C, p}^{-}\left[\begin{array}{c}
\beta_{j} \\
j
\end{array}\right]\left(F_{j}(\mathbf{q}, \mathbf{p}, t) \rho(\mathbf{q}, \mathbf{p}, t)\right)=J(\mathbf{q}, \mathbf{p}, t),
$$

where $\mathbb{D}_{C, q}^{-}\left[\begin{array}{c}\alpha_{j} \\ j\end{array}\right]$ and $\mathbb{D}_{C, p}^{-}\left[\begin{array}{c}\beta_{j} \\ j\end{array}\right]$ are the continuum fractional derivatives with respect to phase-space coordinates $q_{j}$ and $p_{j}$ that are defined by (80) for $0<\alpha_{j}<1$, by Eq. (83) for $\alpha_{j}>1$, and by Eq. (85) for integer odd $\alpha_{j}$ (see Appendix). The functions $\rho(\mathbf{q}, \mathbf{p}, t), V_{j}(\mathbf{q}, \mathbf{p}, t), F(\mathbf{q}, \mathbf{p}, t), J(\mathbf{q}, \mathbf{p}, t)$ are defined by the equations

$$
\begin{aligned}
& \rho(\mathbf{q}, \mathbf{p}, t)=\mathcal{T}_{L \rightarrow C}\left(\rho\left(\mathbf{n}_{q}, \mathbf{n}_{p}, t\right)\right), \quad J(\mathbf{q}, \mathbf{p}, t)=\mathcal{T}_{L \rightarrow C}\left(J\left(\mathbf{n}_{q}, \mathbf{n}_{p}, t\right)\right), \\
& V_{j}(\mathbf{q}, \mathbf{p}, t)=\mathcal{T}_{L \rightarrow C}\left(V_{j}\left(\mathbf{n}_{q}, \mathbf{n}_{p}, t\right)\right), \quad F_{j}(\mathbf{q}, \mathbf{p}, t)=\mathcal{T}_{L \rightarrow C}\left(F_{j}\left(\mathbf{n}_{q}, \mathbf{n}_{p}, t\right)\right) .
\end{aligned}
$$

Proof. Applying the discrete Fourier transform $\mathcal{F}_{\Delta}$ to the first term of the lattice Liouville equation (6), we obtain

$$
\mathcal{F}_{\Delta}\left(\frac{\partial \rho\left(\mathbf{n}_{q}, \mathbf{n}_{p}, t\right)}{\partial t}\right)=\frac{\partial \mathcal{F}_{\Delta}\left(\rho\left(\mathbf{n}_{q}, \mathbf{n}_{p}, t\right)\right)}{\partial t}=\frac{\partial \hat{\rho}\left(\mathbf{k}_{q}, \mathbf{k}_{p}, t\right)}{\partial t},
$$

where

$$
\hat{\rho}\left(\mathbf{k}_{q}, \mathbf{k}_{p}, t\right)=\mathcal{F}_{\Delta}\left(\rho\left(\mathbf{n}_{q}, \mathbf{n}_{p}, t\right)\right) .
$$

Analogously, we can see that

$$
\mathcal{T}_{L \rightarrow C}\left(\frac{\partial \rho\left(\mathbf{n}_{q}, \mathbf{n}_{p}, t\right)}{\partial t}\right)=\frac{\partial \mathcal{T}_{L \rightarrow C}\left(\rho\left(\mathbf{n}_{q}, \mathbf{n}_{p}, t\right)\right)}{\partial t}=\frac{\partial \rho(\mathbf{q}, \mathbf{p}, t)}{\partial t},
$$

where $\rho(\mathbf{q}, \mathbf{p}, t)$ is defined by (22).

The discrete Fourier transform $\mathcal{F}_{\Delta}$ of the second term of (6), gives

$$
\begin{aligned}
\mathcal{F}_{\Delta} & \left(\sum_{j=1}^{3 N} \mathbb{D}_{L}^{-}\left[\begin{array}{c}
\alpha_{j} \\
j
\end{array}\right]\left(V_{j}\left(\mathbf{m}_{q}, \mathbf{m}_{p}, t\right) \rho\left(\mathbf{m}_{q}, \mathbf{m}_{p}, t\right)\right)\right) \\
= & \sum_{j=1}^{3 N}\left(\sum_{n_{j}=-\infty}^{+\infty} \mathrm{e}^{-i k_{j} n_{j} a_{j}} \mathbb{D}_{L}^{-}\left[\begin{array}{c}
\alpha_{j} \\
j
\end{array}\right]\left(V_{j}\left(\mathbf{m}_{q}, \mathbf{m}_{p}, t\right) \rho\left(\mathbf{m}_{q}, \mathbf{m}_{p}, t\right)\right)\right) \\
= & \sum_{j=1}^{3 N} \frac{1}{a_{j}^{\alpha_{j}}}\left(\sum_{n_{j}=-\infty}^{+\infty} \sum_{m_{j}=-\infty}^{+\infty} \mathrm{e}^{-i k_{j} n_{j} a_{j}} K_{\alpha_{j}}^{-}\left(n_{j}-m_{j}\right)\left(V_{j}\left(\mathbf{m}_{q}, \mathbf{m}_{p}, t\right) \rho\left(\mathbf{m}_{q}, \mathbf{m}_{p}, t\right)\right)\right)
\end{aligned}
$$




$$
\begin{aligned}
& =\sum_{j=1}^{3 N} \frac{1}{a_{j}^{\alpha_{j}}}\left(\sum_{n_{j}^{\prime}=-\infty}^{+\infty} \mathrm{e}^{-i k_{j} n_{j}^{\prime} a_{j}} K_{\alpha_{j}}^{-}\left(n_{j}^{\prime}\right) \sum_{m_{j}=-\infty}^{+\infty}\left(V_{k}\left(\mathbf{m}_{q}, \mathbf{m}_{p}, t\right) \rho\left(\mathbf{m}_{q}, \mathbf{m}_{p}, t\right)\right) \mathrm{e}^{-i k_{j} m_{j} a_{j}}\right) \\
& =\sum_{j=1}^{3 N} \frac{1}{a_{j}^{\alpha_{j}}}\left(\hat{K}_{\alpha_{j}}^{-}\left(k_{j} a_{j}\right)\left(\hat{V}_{j}\left(\mathbf{k}_{q}, \mathbf{k}_{p}, t\right) * \hat{\rho}\left(\mathbf{k}_{q}, \mathbf{k}_{p}, t\right)\right)_{q}\right),
\end{aligned}
$$

where $\hat{K}_{\alpha_{j}}^{-}\left(k_{j} a_{j}\right)$ is defined by (8), and $n_{j}^{\prime}=n_{j}-m_{j}$. The symbol $(*)_{q}$ denotes the convolution with respect to $\mathbf{k}_{q}$ that is defined by the equation

$$
\left(\hat{f}\left(\mathbf{k}_{q}, \mathbf{k}_{p}, t\right) * \hat{g}\left(\mathbf{k}_{q}, \mathbf{k}_{p}, t\right)\right)_{q}=\int_{-k_{0} / 2}^{+k_{0} / 2} \prod_{j=1}^{3 N} \mathrm{~d} k_{j}^{\prime} \hat{f}\left(\mathbf{k}_{q}^{\prime}, \mathbf{k}_{p}, t\right) * \hat{g}\left(\mathbf{k}_{q}-\mathbf{k}_{q}^{\prime}, \mathbf{k}_{p}, t\right) .
$$

Similarly, the transform $\mathcal{F}_{\Delta}$ of the third term of (6) gives

$$
\mathcal{F}_{\Delta}\left(\sum_{j=3 N+1}^{6 N} \mathbb{D}_{L}^{-}\left[\begin{array}{c}
\alpha_{j} \\
j
\end{array}\right] \rho\left(\mathbf{m}_{q}, \mathbf{m}_{p}, t\right)\right)=\sum_{j=3 N+1}^{6 N} \frac{1}{a_{j}^{\beta_{j}}}\left(\hat{K}_{\beta_{j}}^{-}\left(k_{j} a_{j}\right)\left(\hat{V}_{j}\left(\mathbf{k}_{q}, \mathbf{k}_{p}, t\right) * \hat{\rho}\left(\mathbf{k}_{q}, \mathbf{k}_{p}, t\right)\right)_{p}\right),
$$

where $(*)_{p}$ denotes the convolution with respect to $\mathbf{k}_{p}$ that is defined by the equation

$$
\left(\hat{f}\left(\mathbf{k}_{q}, \mathbf{k}_{p}, t\right) * \hat{g}\left(\mathbf{k}_{q}, \mathbf{k}_{p}, t\right)\right)_{p}=\int_{-k_{0} / 2}^{+k_{0} / 2} \prod_{j=3 N+1}^{6 N} \mathrm{~d} k_{j}^{\prime} \hat{f}\left(\mathbf{k}_{q}, \mathbf{k}_{p}^{\prime}, t\right) * \hat{g}\left(\mathbf{k}_{q}, \mathbf{k}_{p}-\mathbf{k}_{p}^{\prime}, t\right) .
$$

As a result, the Liouville equation has the form

$$
\begin{aligned}
& \frac{\partial \hat{\rho}\left(\mathbf{k}_{q}, \mathbf{k}_{p}, t\right)}{\partial t}+\sum_{j=1}^{3 N} \hat{K}_{\alpha_{j}}^{-}\left(k_{j} a_{j}\right)\left(\hat{V}_{j}\left(\mathbf{k}_{q}, \mathbf{k}_{p}, t\right) * \hat{\rho}\left(\mathbf{k}_{q}, \mathbf{k}_{p}, t\right)\right)_{q} \\
& +\sum_{j=3 N+1}^{6 N} \hat{K}_{\beta_{j}}^{-}\left(k_{j} a_{j}\right)\left(\hat{F}_{j}\left(\mathbf{k}_{q}, \mathbf{k}_{p}, t\right) * \hat{\rho}\left(\mathbf{k}_{q}, \mathbf{k}_{p}, t\right)\right)_{p}=\hat{J}\left(\mathbf{k}_{q}, \mathbf{k}_{p}, t\right),
\end{aligned}
$$

where the symbols $(*)_{q}$ and $(*)_{p}$ denote the convolution with respect to $\mathbf{k}_{q}$ and $\mathbf{k}_{p}$ respectively.

Then we use

$$
\begin{array}{ll}
\hat{K}_{\alpha_{j}}^{-}\left(a_{j} k_{j}\right)=i \operatorname{sgn}\left(k_{j}\right)\left|a_{j} k_{j}\right|^{\alpha_{j}}, & (j=1, \ldots, 3 N), \\
\hat{K}_{\beta_{j}}^{-}\left(a_{j} k_{j}\right)=i \operatorname{sgn}\left(k_{j}\right)\left|a_{j} k_{j}\right|^{\beta_{j}}, & (j=3 N+1, \ldots, 6 N) .
\end{array}
$$

The limit $a_{j} \rightarrow 0$ gives

$$
\begin{aligned}
& \tilde{K}_{\alpha_{j}}^{-}\left(k_{j}\right)=\lim _{a_{j} \rightarrow 0} \frac{1}{a_{j}^{\alpha_{j}}} \hat{K}_{\alpha_{j}}^{-}\left(k_{j} a_{j}\right)=i k_{j}\left|k_{j}\right|^{\alpha_{j}-1} \quad(j=1, \ldots, 3 N), \\
& \tilde{K}_{\beta_{j}}^{-}\left(k_{j}\right)=\lim _{a_{j} \rightarrow 0} \frac{1}{a_{j}^{\beta_{j}}} \hat{K}_{\beta_{j}}^{-}\left(k_{j} a_{j}\right)=i k_{j}\left|k_{j}\right|^{\beta_{j}-1} \quad(j=3 N+1, \ldots, 6 N) .
\end{aligned}
$$

The "hat"-kernel $\hat{K}_{\alpha_{j}}^{-}\left(k_{j}\right)$ is the discrete Fourier transform $\mathcal{F}_{\Delta}$ of the kernel of the lattice operator. The equation that defines $\hat{K}_{\alpha_{j}}^{-}\left(k_{j}\right)$ has the form

$$
\mathscr{F}_{\Delta}\left(\mathbb{D}_{L}^{-}\left[\begin{array}{c}
\alpha_{j} \\
j
\end{array}\right] f\left(\mathbf{m}_{q}, \mathbf{m}_{p}, t\right)\right)=\frac{1}{a_{j}^{\alpha_{j}}} \hat{K}_{\alpha_{j}}^{-}\left(k_{j} a_{j}\right) \hat{f}\left(\mathbf{k}_{q}, \mathbf{k}_{p}, t\right),
$$

where $\hat{f}\left(\mathbf{k}_{q}, \mathbf{k}_{p}, t\right)=\mathcal{F}_{\Delta}\left\{f\left(\mathbf{m}_{q}, \mathbf{m}_{p}, t\right)\right\}$.

The "tilde"-kernel $\tilde{K}_{\alpha_{j}}^{-}\left(k_{j}\right)$ is the Fourier integral transform $\mathcal{F}$ of the continuum derivative. The equation that defines $\tilde{K}_{\alpha_{j}}^{-}\left(k_{J}\right)$ is

$$
\mathcal{F}\left(\mathbb{D}_{C}^{-}\left[\begin{array}{c}
\alpha_{j} \\
j
\end{array}\right] f(\mathbf{q}, \mathbf{p}, t)\right)=\frac{1}{a_{j}^{\alpha_{j}}} \tilde{K}_{\alpha_{j}}^{-}\left(k_{j} a_{j}\right) \tilde{f}\left(\mathbf{k}_{q}, \mathbf{k}_{p}, t\right),
$$

where $\tilde{f}\left(\mathbf{k}_{q}, \mathbf{k}_{p}, t\right)=\mathcal{F}\{f(\mathbf{q}, \mathbf{p}, t)\}$. 
As a result, the limit $a_{j} \rightarrow 0$ for (31) gives the Liouville equation in the form

$$
\begin{aligned}
& \frac{\partial \tilde{\rho}\left(\mathbf{k}_{q}, \mathbf{k}_{p}, t\right)}{\partial t}+\sum_{j=1}^{3 N} \tilde{K}_{\alpha_{j}}^{-}\left(k_{j}\right)\left(\tilde{V}_{j}\left(\mathbf{k}_{q}, \mathbf{k}_{p}, t\right) * \tilde{\rho}\left(\mathbf{k}_{q}, \mathbf{k}_{p}, t\right)\right)_{q} \\
& +\sum_{j=3 N+1}^{6 N} \tilde{K}_{\beta_{j}}^{-}\left(k_{j}\right)\left(\tilde{F}_{j}\left(\mathbf{k}_{q}, \mathbf{k}_{p}, t\right) * \tilde{\rho}\left(\mathbf{k}_{q}, \mathbf{k}_{p}, t\right)\right)_{p}=\tilde{J}\left(\mathbf{k}_{q}, \mathbf{k}_{p}, t\right),
\end{aligned}
$$

where

$$
\begin{aligned}
& \tilde{K}_{\alpha_{j}}^{-}\left(k_{j}\right)=i k_{j}\left|k_{j}\right|^{\alpha_{j}-1}, \quad(j=1, \ldots, 3 N), \\
& \tilde{K}_{\beta_{j}}^{-}\left(k_{j}\right)=i k_{j}\left|k_{j}\right|^{\beta_{j}-1}, \quad(j=3 N+1, \ldots, 6 N),
\end{aligned}
$$

and

$$
\tilde{\rho}\left(\mathbf{k}_{q}, \mathbf{k}_{p}, t\right)=\operatorname{Lim} \hat{\rho}\left(\mathbf{k}_{q}, \mathbf{k}_{p}, t\right) \quad \tilde{J}\left(\mathbf{k}_{q}, \mathbf{k}_{p}, t\right)=\operatorname{Lim} \hat{J}\left(\mathbf{k}_{q}, \mathbf{k}_{p}, t\right) .
$$

The inverse Fourier transform of (38) gives the fractional Liouville equation (21). As a result, we prove that the lattice fractional Liouville equation (6) is transformed by the operation $\mathcal{T}_{L \rightarrow C}$ into the continuum fractional Liouville equation (21).

This ends the proof.

\section{Fractional Liouville equation for Hamiltonian systems}

For a wide class of physical $N$-particle systems we can use

$$
\begin{aligned}
& V_{j}(\mathbf{q}, \mathbf{p}, t)=V_{j}(\mathbf{p}), \quad(j=1, \ldots, 3 N) \\
& F_{j}(\mathbf{q}, \mathbf{p}, t)=F_{j}(\mathbf{q}, t), \quad(j=3 N+1, \ldots, 6 N) .
\end{aligned}
$$

For Hamiltonian systems with potential forces, we have

$$
\begin{aligned}
& V_{j}(\mathbf{p})=\frac{\partial T(\mathbf{p})}{\partial p_{j}}, \quad(j=1, \ldots, 3 N) \\
& F_{j}(\mathbf{q})=-\frac{\partial U(\mathbf{q})}{\partial q_{j}}, \quad(j=3 N+1, \ldots, 6 N),
\end{aligned}
$$

where the Hamiltonian is $H(\mathbf{q}, \mathbf{p})=T(\mathbf{p})+U(\mathbf{q})$, the function $U(\mathbf{q})$ is the generalized potential of the force, and $T(\mathbf{p})$ is the generalized kinetic energy term. For simple case, we have $V_{j}(\mathbf{p})=p_{j} / M$. Conditions (42) and (43) mean that $V_{j}$ are components of the particle velocity, and $F_{j}$ are components of the force vector that is independent of the particle momenta. In the cases (42) and (43), the fractional Liouville equation for phase-space continuum can be written in the form

$$
\frac{\partial \rho(\mathbf{q}, \mathbf{p}, t)}{\partial t}+\sum_{j=1}^{3 N} V_{j}(\mathbf{p}) \mathbb{D}_{C, q}^{-}\left[\begin{array}{c}
\alpha_{j} \\
j
\end{array}\right] \rho(\mathbf{q}, \mathbf{p}, t)+\sum_{j=3 N+1}^{6 N} F_{j}(\mathbf{q}, t) \mathbb{D}_{C, p}^{-}\left[\begin{array}{c}
\beta_{j} \\
j
\end{array}\right] \rho(\mathbf{q}, \mathbf{p}, t)=J(\mathbf{q}, \mathbf{p}, t) .
$$

As a special case, we also can consider fractional Hamiltonian systems [52,53,13], where

$$
\begin{aligned}
& V_{j}(\mathbf{q}, \mathbf{p}, t)=\mathbb{D}_{C, p}^{-}\left[\begin{array}{c}
\beta_{j+3 N} \\
j+3 N
\end{array}\right] H_{\alpha, \beta}(\mathbf{q}, \mathbf{p}), \quad(j=1, \ldots, 3 N), \\
& F_{j}(\mathbf{q}, \mathbf{p}, t)=-\mathbb{D}_{C, q}^{-}\left[\begin{array}{c}
\alpha_{j-3 N} \\
j-3 N
\end{array}\right] H_{\alpha, \beta}(\mathbf{q}, \mathbf{p}), \quad(j=3 N+1, \ldots, 6 N),
\end{aligned}
$$

where $H_{\alpha, \beta}(\mathbf{q}, \mathbf{p})$ is the generalized Hamiltonian function [52]. In this case, the fractional Liouville equation can be represented in the form

$$
\frac{\partial \rho(\mathbf{q}, \mathbf{p}, t)}{\partial t}+\left\{H_{\alpha, \beta}(\mathbf{q}, \mathbf{p}), \rho(\mathbf{q}, \mathbf{p}, t)\right\}_{\alpha, \beta}=J(\mathbf{q}, \mathbf{p}, t),
$$

where $\{,\}_{\alpha, \beta}$ is the fractional Poisson brackets

$$
\begin{aligned}
\{f(\mathbf{q}, \mathbf{p}), g(\mathbf{q}, \mathbf{p})\}_{\alpha, \beta}= & \sum_{j=1}^{3 N}\left(\mathbb{D}_{C, p}^{-}\left[\begin{array}{c}
\beta_{3 N+j} \\
3 N+j
\end{array}\right] f(\mathbf{q}, \mathbf{p}, t) \mathbb{D}_{c, q}^{-}\left[\begin{array}{c}
\alpha_{j} \\
j
\end{array}\right] g(\mathbf{q}, \mathbf{p}, t)\right. \\
& \left.-\mathbb{D}_{C, q}^{-}\left[\begin{array}{c}
\alpha_{j} \\
j
\end{array}\right] f(\mathbf{q}, \mathbf{p}, t) \mathbb{D}_{c, p}^{-}\left[\begin{array}{c}
\beta_{3 N+j} \\
3 N+j
\end{array}\right] g(\mathbf{q}, \mathbf{p}, t)\right) .
\end{aligned}
$$


If all $\alpha_{j}=1$ and $\beta_{j}=1$, then (50) is the usual Poisson brackets, and (49) is the usual Liouville equation for classical Hamiltonian systems.

If we use $\left(\mathbf{n}_{\mathbf{q}}, \mathbf{n}_{\mathbf{p}}\right)$ instead of $(\mathbf{q}, \mathbf{p})$, and $\mathbb{D}_{L, q}^{-}, \mathbb{D}_{L, p}^{-}$instead of $\mathbb{D}_{C, q}^{-}, \mathbb{D}_{C, p}^{-}$in Eqs. (42)-(50), then we get the correspondent lattice analogs of the considered equations.

\section{Fractional Liouville equation for nonlocal media}

As an example of application of the fractional Liouville equation with conjugate Riesz fractional derivatives, we consider description of non-local plasma-like continuum.

For simplification, we consider an isotropic collisionless nonlocal plasma-like media, where all $\alpha_{j}=\alpha$ and $\beta_{j}=$ $1, J(\mathbf{q}, \mathbf{p}, t)=0$ and $V_{j}(\mathbf{p})=p_{j} / M$. In this case, the $N$-particle distribution function $\rho(\mathbf{q}, \mathbf{p}, t)$ is the product of one-particle reduced distribution function $\rho_{1}(\mathbf{x}, \mathbf{p}, t)$,

$$
\rho(\mathbf{q}, \mathbf{p}, t)=\prod_{s=1}^{N} \rho_{1}\left(\mathbf{x}_{s}, \mathbf{p}_{s}, t\right)
$$

where we use usual space coordinates $\mathbf{x}_{s}=\sum_{j=1}^{3} \mathbf{e}_{j} x_{s j}$ and $\mathbf{p}_{s}=\sum_{j=1}^{3} \mathbf{e}_{j} p_{s j}$ instead of the generalized phase-space coordinates $\mathbf{q}=\left(q_{1}, \ldots, q_{3 N}\right)$ and $\mathbf{p}=\left(p_{1}, \ldots, p_{3 N}\right)$. Here $\mathbf{e}_{j}$ are the basis vectors of the Cartesian coordinate system in $\mathbb{R}^{3}$. The distribution function $\rho_{1}(\mathbf{x}, \mathbf{p}, t)$ describes a probability density to find the particle in the phase volume $d^{3} \mathbf{x} d^{3} \mathbf{p}$.

In this case, the fractional Liouville equation (46) for the one-particle distribution function $\rho_{1}$ takes the form

$$
\frac{\partial \rho}{\partial t}+\sum_{j=1}^{3} V_{j}(\mathbf{p}) \mathbb{D}_{C, x}^{-}\left[\begin{array}{l}
\alpha \\
j
\end{array}\right] \rho_{1}+\sum_{j=1}^{3} F_{3+j}(\mathbf{x}, t) \frac{\partial \rho_{1}}{\partial p_{j}}=0,
$$

where we use

$$
\mathbb{D}_{C, p}^{-}\left[\begin{array}{c}
1 \\
3 N+j
\end{array}\right]=\frac{\partial}{\partial p_{j}} .
$$

Let us apply this Liouville equation with space-fractional derivatives $\mathbb{D}_{C, x}^{-}\left[\begin{array}{l}\alpha \\ j\end{array}\right]$ to describe properties of nonlocal media.

In the absence of the force field, the Liouville equation (52) gives

$$
\frac{\partial \rho}{\partial t}+\sum_{j=1}^{3} V_{j}(\mathbf{p}) \mathbb{D}_{C, x}^{-}\left[\begin{array}{l}
\alpha \\
j
\end{array}\right] \rho=0 .
$$

The solution of this equation will be denoted by $\rho_{0}(\mathbf{x}, \mathbf{p}, t)$. For a weak force field, we can use the charge distribution function in the form

$$
\rho_{1}=\rho_{0}+\delta \rho,
$$

where $\rho_{0}$ is the stationary isotropic homogeneous distribution function unperturbed by the fields, and $\delta \rho$ describes the change of $\rho_{0}$ by the fields. In the linear approximation with respect to field perturbation, we have

$$
\frac{\partial \delta \rho}{\partial t}+\sum_{j=1}^{3}\left(V_{j}(\mathbf{p}) \mathbb{D}_{C, x}^{-}\left[\begin{array}{c}
\alpha \\
j
\end{array}\right] \delta \rho+F_{3+j}(\mathbf{x}, t) \frac{\partial \rho_{0}}{\partial p_{j}}\right)=0 .
$$

If we consider plasma-like media, then the force is the Lorentz force $\mathbf{F}=q \mathbf{E}+q[\mathbf{V}, \mathbf{B}]$, where $q$ is the charge of a particle that moves with velocity $\mathbf{V}=\mathbf{p} / M$ in the presence of an electric field $\mathbf{E}$ and a magnetic field $\mathbf{B}$.

In an isotropic media, the distribution function depends only on the momentum, i.e. $\rho_{0}=\rho_{0}(|\mathbf{p}|)$. In this case, the direction of the vector $\mathbf{e}_{j} \mathbb{D}_{C, x}^{-}\left[\begin{array}{l}\alpha \\ j\end{array}\right] \rho_{0}$ coincides with the vector $\mathbf{p}=M \mathbf{V}$, and its scalar product with $[\mathbf{V}, \mathbf{B}]$ is equal to zero. Therefore, the magnetic field does not affect the distribution function in the linear approximation (56). As a result, we have

$$
\frac{\partial \delta \rho}{\partial t}+\sum_{j=1}^{3}\left(V_{j}(\mathbf{p}) \mathbb{D}_{C, x}^{-}\left[\begin{array}{c}
\alpha \\
j
\end{array}\right] \delta \rho+q E_{j}(\mathbf{x}, t) \frac{\partial \rho_{0}}{\partial p_{j}}\right)=0 .
$$

The Fourier transform with respect to space and time gives

$$
\mathrm{i}\left(\sum_{j=1}^{3} \operatorname{sgn}\left(k_{j}\right)\left|k_{j}\right|^{\alpha} V_{j}-\omega\right) \delta \rho+q \sum_{j=1}^{3}\left(E_{j} \frac{\partial \rho_{0}}{\partial p_{j}}\right)=0 .
$$

If we take the $X$-axis along $\mathbf{k}$, then we have $\mathbf{k}=\left(k_{x}, 0,0\right)$, and

$$
\sum_{j=1}^{3} \operatorname{sgn}\left(k_{j}\right)\left|k_{j}\right|^{\alpha} V_{j}=\left|k_{x}\right|^{\alpha} V_{x},
$$


where $k_{x}>0$ and $\operatorname{sgn}\left(k_{x}\right)=1$, since we use $\mathbf{e}_{x}=\mathbf{k} /|\mathbf{k}|$, and $k_{x}=|\mathbf{k}|$. In this case, Eq. (58) gives

$$
\mathrm{i}\left(\left|k_{x}\right|^{\alpha} V_{x}-\omega\right) \delta \rho+q \sum_{j=1}^{3}\left(E_{j} \frac{\partial \rho_{0}}{\partial p_{j}}\right)=0 .
$$

Then we have

$$
\delta \rho=-\frac{q}{\mathrm{i}\left(\left|k_{x}\right|^{\alpha} V_{x}-\omega\right)} \sum_{j=1}^{3}\left(E_{j} \frac{\partial \rho_{0}}{\partial p_{j}}\right) .
$$

In an unperturbed plasma-like media, the charge density is equal to zero. The charge density that is perturbed by the field is

$$
\rho_{\text {charge }}=q \iiint_{-\infty}^{+\infty} \delta \rho d^{3} \mathbf{p}=\mathrm{i} q^{2} \iiint_{-\infty}^{+\infty} \frac{1}{\left|k_{x}\right|^{\alpha} V_{x}-\omega} \sum_{j=1}^{3}\left(E_{j} \frac{\partial \rho_{0}}{\partial p_{j}}\right) d^{3} \mathbf{p},
$$

where $\rho_{\text {charge }}$ is the bound charge density. The electric polarization vector $\mathbf{P}$ is defined by the relations

$$
\operatorname{div} \mathbf{P}=-\rho_{\text {charge }},
$$

which has the Fourier transform in the form

$$
\mathrm{i}(\mathbf{k}, \mathbf{P})=-\rho_{\text {charge }} .
$$

The polarization $\mathbf{P}$ defines the electric displacement field $\mathbf{D}$ by the equation $\mathbf{D}=\varepsilon_{0} \mathbf{E}+\mathbf{P}$, where $\varepsilon_{0}$ is the electric permittivity. Let the field $\mathbf{E}$ be parallel to $\mathbf{k}$. Then $\mathbf{P}$ be parallel to $\mathbf{k}$, and

$$
\mathbf{P}=\left(\varepsilon_{\|}(|\mathbf{k}|)-\varepsilon_{0}\right) \mathbf{E},
$$

where $\varepsilon_{\|}(|\mathbf{k}|)$ is the longitudinal permittivity. Substitution of (61) and (64) into (63) gives

$$
\left(\varepsilon_{\|}(|\mathbf{k}|)-\varepsilon_{0}\right)(\mathbf{k}, \mathbf{E})=-q^{2} \iiint_{-\infty}^{+\infty} \frac{1}{\left|k_{x}\right|^{\alpha} V_{x}-\omega} \sum_{j=1}^{3}\left(E_{j} \frac{\partial \rho_{0}}{\partial p_{j}}\right) \mathrm{d}^{3} \mathbf{p},
$$

where $(\mathbf{k}, \mathbf{E})$ is the scalar product of vectors $\mathbf{k}$ and $\mathbf{E}$. Since we take the $X$-axis along the vector $\mathbf{k}, \mathbf{E}=\left(E_{x}, 0,0\right)$, and

$$
(\mathbf{k}, \mathbf{E})=k_{x} E_{x}=|\mathbf{k}| E_{x}, \quad \sum_{j=1}^{3}\left(E_{j} \frac{\partial \rho_{0}}{\partial p_{j}}\right)=E_{x} \frac{\partial \rho_{0}}{\partial p_{x}},
$$

where we can use $|\mathbf{k}|$ instead of $k_{x}$.

Using the distribution function

$$
\rho_{0}\left(p_{x}\right)=\iint_{-\infty}^{+\infty} \rho_{0}(|\mathbf{p}|) \mathrm{d} p_{y} \mathrm{~d} p_{z},
$$

we get the following equation to calculate the longitudinal permittivity

$$
\varepsilon_{\|}(|\mathbf{k}|)=\varepsilon_{0}-\frac{q^{2}}{|\mathbf{k}|} \int_{-\infty}^{+\infty} \frac{1}{\left|k_{x}\right|^{\alpha} p_{x} / M-\omega} \frac{\partial \rho_{0}\left(p_{x}\right)}{\partial p_{x}} \mathrm{~d} p_{x},
$$

where $\left|k_{x}\right|=|\mathbf{k}|$ can be used. For isotropic homogeneous case, we can use an equilibrium distribution $\rho_{0}\left(p_{x}\right)$.

Let us consider a plasma-like medium with equilibrium Maxwell's distribution

$$
\rho_{0}\left(p_{x}\right)=\frac{N_{q}}{\sqrt{2 \pi M k_{B} T}} \exp \left(-\frac{p_{x}^{2}}{2 M k_{B} T}\right),
$$

where $k_{B}$ is the Boltzmann constant. Then

$$
\frac{\partial \rho_{0}\left(p_{x}\right)}{\partial p_{x}}=-\frac{2 p_{x} N_{q}}{\sqrt{\pi}\left(2 M k_{B} T\right)^{3 / 2}} \exp \left(-\frac{p_{x}^{2}}{2 M k_{B} T}\right),
$$

where $N_{q}$ is the total number of particles per unit volume.

Using $k_{x}=|\mathbf{k}|$, Eq. (68) can be rewritten in the form

$$
\varepsilon_{\|}(|\mathbf{k}|)=\varepsilon_{0}+\frac{q^{2} N_{q}}{|\mathbf{k}|^{1+\alpha}} \frac{2 M}{\sqrt{\pi}\left(2 M k_{B} T\right)^{3 / 2}} \int_{-\infty}^{+\infty} \frac{p_{x}}{p_{x}-M \omega /|\mathbf{k}|^{\alpha}-\mathrm{i} 0} \exp \left(-\frac{p_{x}^{2}}{2 m k_{B} T}\right) \mathrm{d} p_{x} .
$$


Using new variables

$$
z=\frac{p_{x}}{\sqrt{2 M k_{B} T}}, \quad \xi=\sqrt{\frac{M}{2 k_{B} T}} \cdot \frac{\omega}{|\mathbf{k}|^{\alpha}},
$$

Eq. (71) can be represented in the form

$$
\varepsilon_{\|}(|\mathbf{k}|)=\varepsilon_{0}+\frac{q^{2}}{|\mathbf{k}|^{1+\alpha}} \frac{1}{\sqrt{\pi} k_{B} T} \int_{-\infty}^{+\infty} \frac{z \mathrm{e}^{-z^{2}}}{z-\xi-\mathrm{i} 0} \mathrm{~d} z,
$$

where

$$
\int_{-\infty}^{+\infty} \frac{z \mathrm{e}^{-z^{2}}}{z-\xi-i 0} \mathrm{~d} z=\sqrt{\pi}+P . V . \int_{-\infty}^{+\infty} \frac{\xi \mathrm{e}^{-z^{2}}}{z-\xi} \mathrm{d} z+\mathrm{i} \pi \xi \mathrm{e}^{-\xi^{2}} .
$$

It should be noted that $\mathbf{k}, \mathbf{x}, k_{x}$ and $x_{j}$ are dimensionless variables.

We consider Eq. (73) for two cases that are characterized by the large and small values of the variable $\xi$.

(1) For small values $\xi \ll 1$, we have

$$
\begin{aligned}
P . V . \int_{-\infty}^{+\infty} \frac{x \mathrm{e}^{-z^{2}}}{z-\xi} \mathrm{d} z & =P . V . \int_{-\infty}^{+\infty} \frac{\xi \mathrm{e}^{-(y+\xi)^{2}}}{y} \mathrm{~d} y \\
& =P . V . \int_{-\infty}^{+\infty} \mathrm{e}^{-y^{2}}\left(y^{-1} \xi-2 \xi^{2}-\left(y^{-1}+2 y\right) \xi^{3}+\left(2-(4 / 3) y^{2}\right) \xi^{4}+\cdots\right) \mathrm{d} y \\
& =-2 \sqrt{\pi} \xi^{2}+\sqrt{\pi} \xi^{4}+\cdots \quad(\xi \ll 1),
\end{aligned}
$$

where $y=z-\xi$, and we take into account that the integrals of the odd terms in $y$ are zero. Substitution of (75) and (74) into (73) gives

$$
\varepsilon_{\|}(|\mathbf{k}|)=\varepsilon_{0}+\frac{q^{2} N_{q}}{k_{B} T|\mathbf{k}|^{1+\alpha}}-\frac{q^{2} N_{q} M \omega^{2}}{k_{B}^{2} T^{2}|\mathbf{k}|^{3 \alpha+1}}+\frac{q^{2} N_{q} M^{2} \omega^{4}}{4 k_{B}^{3} T^{3}|\mathbf{k}|^{5 \alpha+1}}+\cdots .
$$

In the case $\xi \ll 1$, the imaginary part of the permittivity $\varepsilon_{\|}(|\mathbf{k}|)$ is relatively small but it is not exponentially small, because of the smallness of the phase volume, where the condition $|\mathbf{k}|^{\alpha} p_{x} / M-\omega=0$ holds.

(2) For large values $\xi \gg 1$, we have

$$
\begin{aligned}
P . V . \int_{-\infty}^{+\infty} \frac{x \mathrm{e}^{-z^{2}}}{z-\xi} \mathrm{d} z & =-\int_{-\infty}^{+\infty} \frac{\mathrm{e}^{-z^{2}}}{1-z / \xi} \mathrm{d} z=-\int_{-\infty}^{+\infty} \mathrm{e}^{-z^{2}}\left(1+\sum_{m=1}^{\infty}\left(\frac{z}{\xi}\right)^{m}\right) \mathrm{d} z \\
& =-\sqrt{\pi}-\frac{\sqrt{\pi}}{2 \xi^{2}}-\frac{3 \sqrt{\pi}}{4 \xi^{4}}-\cdots \quad(\xi \gg 1),
\end{aligned}
$$

where we take into account that the integrals of the odd terms in $z$ are zero also. Substituting (77) and (74) into (73), we get

$$
\varepsilon_{\|}(|\mathbf{k}|)=\varepsilon_{0}-\frac{q^{2} N_{q}}{M \omega^{2}}|\mathbf{k}|^{\alpha-1}-\frac{3 q^{2} N_{q} k_{B} T}{M^{2} \omega^{4}}|\mathbf{k}|^{3 \alpha-1}+\cdots .
$$

For Maxwell's distribution, an exponentially small part of the charged particles has the velocity $V_{x}=\omega /|\mathbf{k}| \gg V_{T}=$ $\sqrt{k_{B} T / m}$, where $V_{T}$ is the average velocity of charged particles. Therefore the imaginary part of the permittivity $\varepsilon_{\|}(|\mathbf{k}|)$ is exponentially small.

Eqs. (76) and (78) can be used to obtain the scalar potentials of electric field for the fractional nonlocal plasma-like media $[17,24]$.

\section{Conclusion}

In this paper, the Liouville equation for unbounded homogeneous phase-space lattice with long-range jumps is suggested. Using the methods proposed in Refs. [25-27], we prove that the continuous limit transforms the suggested Liouville equation for lattice phase-space into the fractional nonlocal Liouville equation for continuum phase-space. This fractional Liouville equation contains the generalized conjugate Riesz derivatives on non-integer orders with respect to phase-space coordinates. As an example, we consider an application of the fractional Liouville equation with the Riesz derivatives of non-integer orders to describe properties of plasma-like nonlocal media. 
We assume that the lattice fractional Fokker-Planck equation, which is suggested in Ref. [38], can be obtained from the phase-space lattice Liouville equation that is proposed in this paper. The lattice Bogoliubov hierarchy equations can be easily derived from the suggested lattice Liouville equation by using the reduced distribution functions. The corresponding lattice hydrodynamic approximation can also be obtained from the lattice Bogoliubov hierarchy equations. It allows us to formulate fractional-nonlocal statistical mechanics $[21,13]$ on the lattice phase-space. The suggested lattice-continuum transform operator allows us to have a correspondence between lattice fractional statistical mechanics and fractional nonlocal statistical theory.

The fractional Bogoliubov hierarchy equations with derivatives of non-integer orders with respect to phase-space coordinates can be obtained from the suggested fractional Liouville equation with Riesz fractional derivatives in the same way as was done for the Liouville equation with Caputo derivatives in Refs. [21,22]. The Vlasov equation and the Fokker-Planck equation with the fractional derivatives of the Riesz type can be also obtained from fractional Liouville equations [38]. All these equations form a basis of fractional nonlocal statistical mechanics.

\section{Appendix. Continuum fractional derivative of the Riesz type}

Let us consider the continuum fractional derivative $\mathbb{D}_{C}^{-}\left[\begin{array}{c}\alpha_{j} \\ j\end{array}\right]$ of the Riesz type that has the property

$$
\mathcal{F}\left(\mathbb{D}_{C}^{-}\left[\begin{array}{c}
\alpha_{j} \\
j
\end{array}\right] f(\mathbf{x})\right)(\mathbf{k})=i \operatorname{sgn}\left(k_{j}\right)\left|k_{j}\right|^{\alpha_{j}}(\mathcal{F} f)(\mathbf{k}) \quad\left(\alpha_{j}>0\right) .
$$

For $0<\alpha_{j}<1$ the operator $\mathbb{D}_{C}^{-}\left[\begin{array}{c}\alpha_{j} \\ j\end{array}\right]$ can be considered as the conjugate Riesz derivative [6] with respect to $x_{j}$. Therefore, the operator (79) can be called a generalized conjugate derivative of the Riesz type.

For $0<\alpha_{j}<1$ the fractional operator (79) can be defined by the equation

$$
\mathbb{D}_{C}^{-}\left[\begin{array}{c}
\alpha_{j} \\
j
\end{array}\right] f(\mathbf{x})=\frac{\partial}{\partial x_{j}} \int_{\mathbb{R}^{1}} R_{1-\alpha_{j}}\left(x_{j}-z_{j}\right) f\left(\mathbf{x}+\left(z_{j}-x_{j}\right) \mathbf{e}_{j}\right) \mathrm{d} z_{j}, \quad\left(0<\alpha_{j}<1\right),
$$

where $\mathbf{e}_{j}$ is the basis of the Cartesian coordinate system. The function $R_{\alpha_{j}}(x)$ is the Riesz kernel, that is defined by

$$
R_{\alpha_{j}}(x)= \begin{cases}\gamma_{1}^{-1}\left(\alpha_{j}\right)|x|^{\alpha_{j}-1} & \alpha_{j} \neq 2 n+1, n \in \mathbb{N} \\ -\gamma_{1}^{-1}\left(\alpha_{j}\right)|x|^{\alpha_{j}-1} \ln |x| & \alpha_{j}=2 n+1, n \in \mathbb{N}\end{cases}
$$

The constant $\gamma_{1}\left(\alpha_{j}\right)$ has the form

$$
\gamma_{1}\left(\alpha_{j}\right)= \begin{cases}2^{\alpha_{j}} \pi^{1 / 2} \Gamma(\alpha / 2) / \Gamma\left(\left(1-\alpha_{j}\right) / 2\right) & \alpha_{j} \neq 2 n+1, \\ (-1)^{\left(1-\alpha_{j}\right) / 2} 2^{\alpha_{j}-1} \pi^{1 / 2} \Gamma(\alpha / 2) \Gamma\left(1+\left[\alpha_{j}-1\right] / 2\right) & \alpha_{j}=2 n+1,\end{cases}
$$

where $N \in \mathbb{N}$ and $\alpha_{j} \in \mathbb{R}_{+}$. Note the distinction between the continuum fractional derivatives $\mathbb{D}_{C}^{-}\left[\begin{array}{c}\alpha_{j} \\ j\end{array}\right]$ and the Riesz potential consists in the use of $\left|k_{j}\right|^{-\alpha_{j}}$ instead of $|\mathbf{k}|^{-\alpha_{j}}$.

For $\alpha_{j}>1$ the fractional operator (79) can be defined by the equation

$$
\mathbb{D}_{C}^{-}\left[\begin{array}{c}
\alpha_{j} \\
j
\end{array}\right] f(\mathbf{x})=\frac{1}{d_{1}\left(m, \alpha_{j}-1\right)} \frac{\partial}{\partial x_{j}} \int_{\mathbb{R}^{1}} \frac{1}{\left|z_{j}\right|^{\alpha_{j}}}\left(\Delta_{z_{j}}^{m} f\right)(\mathbf{x}) \mathrm{d} z_{j}, \quad\left(1<\alpha_{j}<m+1\right),
$$

where $\left(\Delta_{z_{j}}^{m} u\right)(\mathbf{x})$ is a finite difference of order $m$ of a function $f(\mathbf{x})$ with the vector step $\mathbf{z}_{j}=z_{j} \mathbf{e}_{j} \in \mathbb{R}^{N}$ for the point $\mathbf{x}=\sum_{j=1}^{N} x_{j} \mathbf{e}_{j} \in \mathbb{R}^{N}$. The centered difference

$$
\left(\Delta_{z_{j}}^{m} f\right)\left(\mathbf{x}_{j}\right)=\sum_{n=0}^{m}(-1)^{n} \frac{m !}{n !(m-n) !} f\left(\mathbf{x}-(m / 2-n) z_{j} \mathbf{e}_{j}\right) .
$$

The constant $d_{1}\left(m, \alpha_{j}\right)$ is defined by

$$
d_{1}\left(m, \alpha_{j}\right)=\frac{\pi^{3 / 2} A_{m}\left(\alpha_{j}\right)}{2^{\alpha_{j}} \Gamma(1+\alpha / 2) \Gamma\left(\left(1+\alpha_{j}\right) / 2\right) \sin (\pi \alpha / 2)},
$$

where

$$
A_{m}\left(\alpha_{j}\right)=2 \sum_{s=0}^{[m / 2]}(-1)^{s-1} \frac{m !}{s !(m-s) !}(m / 2-s)^{\alpha_{j}}
$$

for the centered difference (84). The constant $d_{1}\left(m, \alpha_{j}\right)$ is different from zero for all $\alpha_{j}>0$ in the case of an even $m$ and centered difference $\left(\Delta_{i}^{m} u\right)$ (see Theorem 26.1 in Ref. [3]). Note that the derivative (83) does not depend on the choice of $m>\alpha_{j}-1$. Therefore, we can always choose an even number $m$ so that it is greater than $\alpha_{1}-1$, and then we can use the centered difference (84) for all positive real values of $\alpha_{j}$. 
For integer odd values of $\alpha_{j}$, we have

$$
\mathbb{D}_{C}^{-}\left[\begin{array}{c}
2 m+1 \\
j
\end{array}\right] f(\mathbf{x})=(-1)^{m} \frac{\partial^{2 m+1} f(\mathbf{x})}{\partial x_{j}^{2 m+1}}, \quad(m \in \mathbb{N}) .
$$

Eq. (85) means that the fractional derivatives $\mathbb{D}_{C}^{-}\left[\begin{array}{c}\alpha_{j} \\ j\end{array}\right]$ of the odd orders $\alpha_{j}$ are local operators represented by the usual derivatives of integer orders. Note that the continuum derivative $\mathbb{D}_{C}^{-}\left[\begin{array}{c}2 m \\ j\end{array}\right]$, where $m \in \mathbb{N}$, cannot be considered as a usual local derivative $\partial^{2 m} / \partial x_{j}^{2 m}$. of the order $2 m$ with respect to $x_{j}$.

\section{References}

[1] A.A. Vlasov, Statistical Distribution Functions, Nauka, Moscow, 1966, 356p (in Russian).

[2] A.A. Vlasov, Nonlocal Statistical Mechanics, Nauka, Moscow, 1978, 264p (in Russian).

[3] S.G. Samko, A.A. Kilbas, O.I. Marichev, Fractional Integrals and Derivatives Theory and Applications, Gordon and Breach, New York, 1993, 1006p.

[4] I. Podlubny, Fractional Differential Equations: An Introduction to Fractional Derivatives, Fractional Differential Equations, to Methods of Their Solution and Some of Their Applications, Academic Press, San Diego, 1998, 340p.

[5] A.A. Kilbas, H.M. Srivastava, J.J. Trujillo, Theory and Applications of Fractional Differential Equations, Elsevier, Amsterdam, 2006, 353p.

[6] V.V. Uchaikin, Fractional Derivatives for Physicists and Engineers. Volume I. Background and Theory, Springer, Higher Education Press, 2012, 385p.

[7] F. Mainardi, Fractional calculus: Some basic problems in continuum and statistical mechanics, in: A. Carpinteri, F. Mainardi (Eds.), Fractals and Fractional Calculus in Continuum Mechanics, Springer, Wien and New York, 1997, pp. 291-348. arXiv:1201.0863.

[8] R.E. Gutierrez, J.M. Rosario, J.A. Tenreiro Machado, Fractional order calculus: basic concepts and engineering applications, Math. Probl. Eng. 2010 (2010) 375858 .

[9] D. Valerio, J.J. Trujillo, M. Rivero, J.A. Tenreiro Machado, D. Baleanu, Fractional calculus: A survey of useful formulas, Eur. Phys. J. Spec. Top. 222 (8) (2013) 1827-1846.

[10] J. Sabatier, O.P. Agrawal, J.A. Tenreiro Machado (Eds.), Advances in Fractional Calculus. Theoretical Developments and Applications in Physics and Engineering, Springer, Dordrecht, 2007, 552p.

[11] A.C.J. Luo, V.S. Afraimovich (Eds.), Long-range Interaction, Stochasticity and Fractional Dynamics, Springer, Berlin, 2010, 311p.

[12] F. Mainardi, Fractional Calculus and Waves in Linear Viscoelasticity: An Introduction to Mathematical Models, World Scientific, Singapore, 2010, 368p.

[13] V.E. Tarasov, Fractional Dynamics: Applications of Fractional Calculus to Dynamics of Particles, Fields and Media, Springer, New York, 2011, 450p.

[14] J. Klafter, S.C. Lim, R. Metzler (Eds.), Fractional Dynamics. Recent Advances, World Scientific, Singapore, 2011.

[15] V.E. Tarasov, Review of some promising fractional physical models, Internat. J. Modern Phys. B 27 (9) (2013) 1330005.

[16] V. Uchaikin, R. Sibatov, Fractional Kinetics in Solids: Anomalous Charge Transport in Semiconductors, Dielectrics and Nanosystems, World Scientific, Singapore, 2013, 276p.

[17] V.E. Tarasov, Fractional generalization of Liouville equations, Chaos 14 (1) (2004) 123-127. arXiv:nlin.CD/0312044.

[18] V.E. Tarasov, Fractional systems and fractional Bogoliubov hierarchy equations, Phys. Rev. E 71 (1) (2005) 011102.12 pages. arXiv:cond-mat/0505720.

[19] V.E. Tarasov, Fractional Liouville and BBGKI equations, J. Phys.: Conf. Ser. 7 (2005) 17-33. arXiv:nlin.CD/0602062.

[20] V.E. Tarasov, Transport equations from Liouville equations for fractional systems, Internat. J. Modern Phys. B 20 (3) (2006) 341-353. arXiv:condmat/0604058.

[21] V.E. Tarasov, Fractional statistical mechanics, Chaos 16 (3) (2006) 033108. 7 pages. arXiv:0710.1807.

[22] V.E. Tarasov, Liouville and Bogoliubov equations with fractional derivatives, Modern Phys. Lett. B 21 (5) (2007) 237-248. arXiv:0711.0859.

[23] V.E. Tarasov, Power-law spatial dispersion from fractional Liouville equation, Phys. Plasmas 20 (10) (2013) 102110.10 pages. arXiv:1307.4930.

[24] V.E. Tarasov, J.J. Trujillo, Fractional power-law spatial dispersion in electrodynamics, Ann. Phys. 334 (2013) 1-23.

[25] V.E. Tarasov, Continuous limit of discrete systems with long-range interaction, J. Phys. A 39 (48) (2006) 14895-14910. arXiv:0711.0826.

[26] V.E. Tarasov, Map of discrete system into continuous, J. Math. Phys. 47 (9) (2006) 092901. arXiv:0711.2612.

[27] V.E. Tarasov, Toward lattice fractional vector calculus, J. Phys. A 47 (35) (2014) 355204.51 pages.

[28] V.E. Tarasov, G.M. Zaslavsky, Fractional dynamics of coupled oscillators with long-range interaction, Chaos 16 (2) (2006) 023110.

[29] V.E. Tarasov, G.M. Zaslavsky, Fractional dynamics of systems with long-range interaction, Commun. Nonlinear Sci. Numer. Simul. 11 (8) (2006) 885-898. arXiv:1107.5436.

[30] N. Laskin, G. Zaslavsky, Nonlinear fractional dynamics on a lattice with long range interactions, Physica A 368 (1) (2006) $38-54$. arXiv:nlin/0512010.

[31] R. Ishiwata, Y. Sugiyama, Relationships between power-law long-range interactions and fractional mechanics, Physica A 391 (23) (2012) 5827-5838.

[32] V.E. Tarasov, Lattice model with power-law spatial dispersion for fractional elasticity, Cent. Eur. J. Phys. 11 (11) (2013) $1580-1588$.

[33] V.E. Tarasov, Lattice model of fractional gradient and integral elasticity: Long-range interaction of Grünwald-Letnikov-Riesz type, Mech. Mater. 70 (1) (2014) 106-114.

[34] V.E. Tarasov, Fractional gradient elasticity from spatial dispersion law, ISRN Condensed Matter Physics 2014 (2014) 794097.13 pages. arXiv:1306.2572.

[35] V.E. Tarasov, Lattice with long-range interaction of power-law type for fractional non-local elasticity, Int. J. Solids Struct. 51 (15-16) (2014) $2900-2907$.

[36] V.E. Tarasov, Non-linear fractional field equations: weak non-linearity at power-law non-locality, Nonlinear Dynam. (2014) http://dx.doi.org/10.1007/s11071-014-1342-0.

[37] V.E. Tarasov, Fractional quantum field theory: From lattice to continuum, Adv. High Energy Phys. 2014 (2014) 957863.14 pages.

[38] V.E. Tarasov, Large lattice fractional Fokker-Planck equation, J. Stat. Mech. Theory Exp. 2014 (9) (2014) P09036.

[39] A. Campa, T. Dauxois, S. Ruffo, Statistical mechanics and dynamics of solvable models with long-range interactions, Phys. Rep. 480 (3-6) (2009) 57-159. arXiv:0907.0323.

[40] J. Barre, F. Bouchet, T. Dauxois, S. Ruffo, Large deviation techniques applied to systems with long-range interactions, J. Stat. Phys. 119 (3-4) (2005) 677-713. arXiv:cond-mat/0406358.

[41] S. Ruffo, Equilibrium and nonequilibrium properties of systems with long-range interactions, Eur. Phys. J. B 64 (3-4) (2008) 355-363.

[42] C. Nardini, S. Gupta, S. Ruffo, T. Dauxois, F. Bouchet, Kinetic theory for non-equilibrium stationary states in long-range interacting systems, J. Stat. Mech. 2012 (1) (2012) L01002. arXiv:1111.6833.

[43] Y. Levin, R. Pakter, F.B. Rizzato, T.N. Teles, F.P.C. Benetti, Nonequilibrium statistical mechanics of systems with long-range interactions, Phys. Rep. 535 (1) (2014) 1-60. arXiv:1310.1078

[44] H. Hinrichsen, Non-equilibrium phase transitions with long-range interactions, J. Stat. Mech. 2007 (7) (2007) P07006. arXiv:cond-mat/0702169.

[45] R. Bachelard, C. Chandre, D. Fanelli, X. Leoncini, S. Ruffo, Abundance of regular orbits and nonequilibrium phase transitions in the thermodynamic limit for long-range systems, Phys. Rev. Lett. 101 (26) (2008) 260603.

[46] R.L. Liboff, Kinetic Theory: Classical, Quantum and Relativistic Description, second ed., Wiley, New York, 1998, p. 548.

[47] J.W. Gibbs, Elementary Principles in Statistical Mechanics, Scribner, New York, 1905. 
[48] V.E. Tarasov, Classical non-Hamiltonian dynamics, in: Quantum Mechanics of Non-Hamiltonian and Dissipative Systems, Elsevier Science, 2008 (Chapter 16).

[49] M. Born, Is classical mechanics in fact deterministic? Phys. Bl. 9 (1955) 49-54; Physics in My Generation, Springer, New York, 1968, pp. $78-83$.

[50] A. Erdelyi, W. Magnus, F. Oberhettinger, F.G. Tricomi, Higher Transcendental Functions, Vol. 1, McGraw-Hill, New York, 1953, and (Krieeger, Melbourne, Florida, 1981).

[51] V.E. Tarasov, No violation of the Leibniz rule. no fractional derivative, Commun. Nonlinear Sci. Numer. Simul. 18 (11) (2013) $2945-2948$.

[52] V.E. Tarasov, Fractional generalization of gradient and Hamiltonian systems, J. Phys. A 38 (26) (2005) 5929-5943.

[53] V.E. Tarasov, Fractional generalization of gradient systems, Lett. Math. Phys. 73 (1) (2005) 49-58. arXiv:nlin.CD/0604007. 